\title{
Equal Intensity Contours for Whole-Body Vibrations Compared With Vibrations Cross-Modally Matched to Isophones
}

\author{
Sebastian Merchel, M. Ercan Altinsoy and Maik Stamm \\ Chair of Communication Acoustics, Dresden University of Technology, Germany \\ sebastian.merchel@tu-dresden.de
}

\begin{abstract}
In this study, two experiments were conducted to determine the curves of equal intensity perception for sinusoidal vertical whole-body vibrations (WBV) of seated subjects over the frequency range from $10 \mathrm{~Hz}$ to $250 \mathrm{~Hz}$. Vibrations were presented to subjects using a flat hard seat. In total, 10 participants were asked to match the intensity of different vibrations, using a method of adjustment. The obtained contours were compared with the threshold of vibration and to vibrations cross-modally matched to tones from isophones.

The shapes of the equal intensity contours in the present study show reasonable agreement with the contours from other studies despite the use of different methodologies and experimental questions. The contours show a characteristic similar to the perception threshold. No dependency of vibration magnitude on the shape of the contours was found in the applied dynamic range. However, large inter-individual variations were observed. The results imply that vibration curves that are cross-modally matched to isophones show similar characteristics.
\end{abstract}

Keywords: Equal Intensity Contour, Whole-Body Vibration, Isophone, AudioTactile Perception, Cross-Modality Matching

\section{Introduction}

In a previous study [1], pure tones from isophones were cross-modally matched to vertical sinusoidal whole-body vibrations. It was hypothesized that the resulting vibrations are perceived with equal intensity. The present study investigates whether those contours coincide with curves of equal intensity perception for vertical sinusoidal whole-body vibrations.

Earlier studies have determined equivalent comfort contours for vertical sinusoidal whole-body vibrations mainly in the context of health risk estimation. Thus, only high vibration magnitudes and low frequencies have been investigated. A review can be found in Griffin [2]. The overall shape of the equivalent comfort contours is somewhat consistent between most of these studies: above 
$5 \mathrm{~Hz}$ the contours rise with increasing frequency. However, these contours represent discomfort or annoyance from whole-body vibrations. It is questionable if (dis-)comfort or annoyance can be compared to perceived vibration intensity. Few studies exist that measure vibrations close to the perception threshold or for frequencies above $100 \mathrm{~Hz}$. Therefore, equal intensity contours will be measured for low magnitudes between $10 \mathrm{~Hz}$ and $250 \mathrm{~Hz}$.

In an aim to compare the equal intensity contours with the perception threshold for sinusoidal vertical whole-body vibrations, threshold curves from various laboratories $[3-8]$ were obtained and are summarized in Figure 1. The shapes of the threshold contours are similar. An overall trend of increasing threshold with increasing frequency is found over the range from $5 \mathrm{~Hz}$ to $300 \mathrm{~Hz}$. However, Miwa [3] and Parson \& Griffin [4] measured lower thresholds, particularly at lower frequencies. Different body postures or body support might explain some variability between studies in this frequency range. For example, the surface of the seat used by Morioka \& Griffin [5] was contoured (to provide contact with the ischial tuberosities) and approximately half the size of the flat seats used in other studies, which themselves also provided contact with the thighs. The absence of contact with the thighs might reduce sensitivity to low-frequency vibrations. Differences between studies might also be partially explained by different psycho-physical methods. For example, Miwa [3], Bellmann [6], Stamm et al. [7] and Merchel \& Leppin [8] used different adaptive n-interval forced choice methods, whereas Parsons \& Griffin [4] and Morioka \& Griffin [5] employed a 'yes-no' method.

Two frequency regions can be separated and fitted by 1st order regressions. At lower frequencies, the perception threshold increases slightly by approximately $1.3 \mathrm{~dB}$ per octave. Above $150 \mathrm{~Hz}$, the increase climbs up to $6.5 \mathrm{~dB}$ per octave. No prediction can be made outside of the shown frequency range. The regression curves must be interpreted carefully since few measurement points are available at higher frequencies. However, Morioka and Griffin [5] reported a significant increase for frequencies above $200 \mathrm{~Hz}$. It can be seen that the threshold curve from ISO 2631:1989 [9], which was removed from the revised ISO 2631:2003 [10], does not represent the data well. Thus, the fitted curves will be used for comparison later in this study.

\section{Experiment}

Two experiments were conducted to determine the curves of equal intensity perception of sinusoidal vertical whole-body vibrations for seated subjects. The subjects were asked to compare the intensity of a reference vibration with a test stimulus using a method of adjustment. In the first experiment, two curves determined using different reference frequencies were obtained. One reference frequency was selected and used in the second experiment to determine equal intensity curves over a wider dynamic range. 


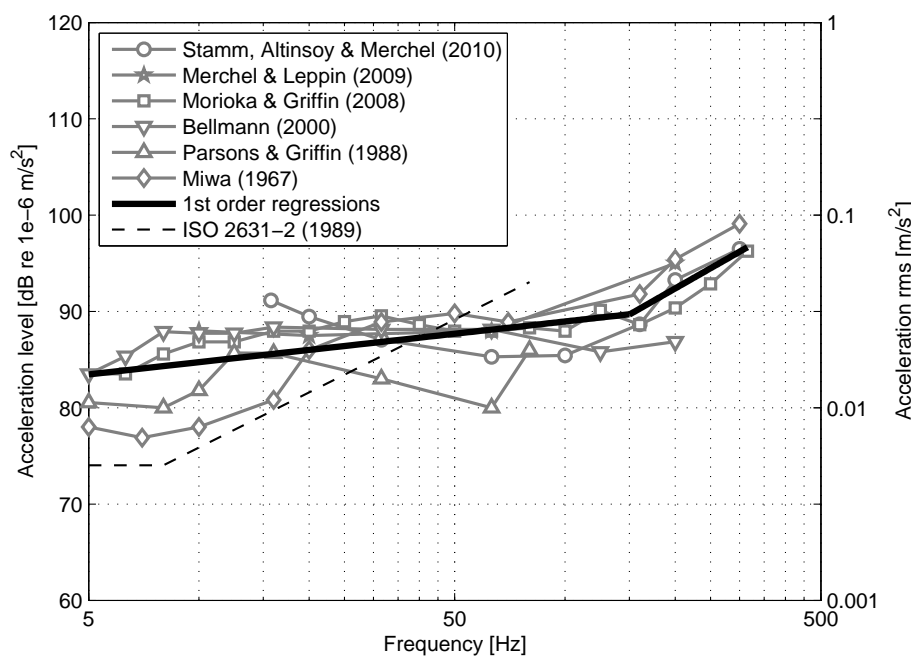

Fig. 1. Perception threshold for vertical sinusoidal whole-body vibrations from various laboratories in comparison to the threshold from ISO 2631:1989 [9]. 1st order regressions were fitted to the data below and above $150 \mathrm{~Hz}$.

\subsection{Setup}

Whole-body vibrations were generated vertically using an electro-dynamic shaker. The subject was seated on a flat hard wooden seat $(460 \mathrm{~mm} \times 460 \mathrm{~mm})$ with both feet on the ground. There was no backrest. The transfer characteristic of the vibrating chair is strongly dependent on the individual person [11]. This phenomenon is referred to as the body related transfer function (BRTF). The BRTF of each subject was individually monitored using a vibration pad (B\&K Type 4515B) and a Sinus Harmonie quadro measuring board and individually compensated using inverse filters in Matlab.

For higher frequencies, the vibration chair can emit acoustic noise. Pink noise was presented at $74 \mathrm{~dB}(\mathrm{~A})$ to acoustically mask the noises emitted by the chair. In addition, unwanted auditory feedback possibly delivered through bone conduction will be masked as well. The audio signal was delivered through an external Hammerfall DSP Multiface sound card, amplified by a Phone-Amp G93 and reproduced through a set of Sennheiser HDA 200 closed dynamic headphones.

The participant was able to control the amplitude of the vibration using a rotary knob that was infinitely adjustable and did not possess any visual indicators (Griffin Technology, PowerMate). 


\subsection{Subjects}

Ten subjects ( 6 male and 4 female) voluntarily participated in both experiments. Most of the participants were students between 19 years and 27 years old (mean 23 years). The participants had masses between $62 \mathrm{~kg}$ and $85 \mathrm{~kg}$ (mean $71 \mathrm{~kg}$ ) and indicated to have no hearing or spinal damage.

The subjects were instructed to sit upright with comfortable posture, their hands on their thighs and both feet flat on the ground. Additional plates were used to adjust the height of the feet until the thighs were approximately horizontal and level with the seat.

\subsection{Stimuli and Experimental Design}

Seven vibration frequencies over a wide frequency range $(10 \mathrm{~Hz}, 20 \mathrm{~Hz}, 50 \mathrm{~Hz}$, $100 \mathrm{~Hz}, 150 \mathrm{~Hz}, 200 \mathrm{~Hz}$ and $250 \mathrm{~Hz}$ ) were selected for the experiments. In the first experiment, two frequencies were selected as reference frequencies $(20 \mathrm{~Hz}$ and $100 \mathrm{~Hz}$ ) with a fixed acceleration level of $110 \mathrm{~dB}$. The reference vibration was presented for one second followed by a $0.5 \mathrm{~s}$ break. Afterwards, one of the test frequencies was reproduced for one second. The order of the test frequencies was completely randomized. All vibration signals were faded in and out using half a hanning window of $50 \mathrm{~ms}$ flanks. Reference and test stimuli were marked visually using the experimental interface controlled by Matlab. The task of the subject was to adaptively adjust the perceived intensity of the test vibration to the perceived intensity of the reference vibration using the rotary knob. The test frequency was adjustable with a minimum step size of $0.25 \mathrm{~dB}$. The initial acceleration level of the test whole-body vibration was $90 \pm 5 \mathrm{~dB}$ (a random offset was used for each trial). A low initial acceleration was necessary because the dynamic range of vibration perception is small and a high level of vibration may cause discomfort. This sequence was automatically repeated until the participant was satisfied with his/her match. The subject was free to take as much time as necessary to make the proper adjustments. The total duration of the experiment varied between participants and took between 25 and 35 minutes to match both reference vibrations with all 7 test vibrations (including a familiarization phase in the beginning). For the $100 \mathrm{~Hz}$ reference condition, each match was repeated three times for all participants to check intra-individual repeatability.

In the second experiment, a reference frequency of $20 \mathrm{~Hz}$ was used with various acceleration levels ranging close to the perceptual threshold until moderately strong values $(100 \mathrm{~dB}, 105 \mathrm{~dB}, 110 \mathrm{~dB}, 115 \mathrm{~dB}$ and $120 \mathrm{~dB})$. The experimental design was identical to the first experiment. The total duration of the experiment varied between 35 minutes and 45 minutes, depending on the individual subject. 


\section{Results and Discussion}

\subsection{Experiment 1 - Different Reference Frequencies}

Figure 2 shows the individual equal intensity contours for vertical sinusoidal whole-body vibrations for all subjects using a reference frequency of $100 \mathrm{~Hz}$ with an acceleration level of $110 \mathrm{~dB}$. As expected, the results show small deviations at the reference frequency. The individual contours match well toward higher frequencies, but increasing inter-individual differences are found toward lower frequencies. The intra-individual standard deviations (not plotted here) are comparatively small, in the range between $1 \mathrm{~dB}$ and $3 \mathrm{~dB}$ independent of frequency, which indicates good repeatability.

In Figure 3, individual equal intensity contours for a reference frequency of $20 \mathrm{~Hz}$ with an acceleration level of $110 \mathrm{~dB}$ are plotted. The measured individual contours agree for low frequencies but show considerable differences toward higher frequencies. The amount of variation is comparable to the data shown in Figure 2 . It can be concluded that contours of equally perceived intensity are highly subject dependent. This dependency could be explained with strong inter-individual differences in the perception threshold as found in [8]. However, no threshold data exist for the participants in the present study.

To compare the results, the mean was calculated for both conditions. The averaged intensity contours are plotted in Figure 4 with inter-individual standard deviations. The mean values increase depending on the frequency from $10 \mathrm{~Hz}$ to $250 \mathrm{~Hz}$. The inter-individual standard deviations increase with increasing distance from the reference frequencies. However, the averaged contours show reasonable agreement. Thus, the $20 \mathrm{~Hz}$ reference stimulus was used with various levels in the following experiment. The averaged contours should be interpreted and used carefully because of the high inter-individual deviations.

\subsection{Experiment 2 - Different Magnitudes}

Figure 5 shows the averaged equal intensity contours for vertical sinusoidal whole-body vibrations using a reference frequency of $20 \mathrm{~Hz}$ with acceleration levels of $100 \mathrm{~dB}, 105 \mathrm{~dB}, 110 \mathrm{~dB}, 115 \mathrm{~dB}$ and $120 \mathrm{~dB}$. The contours are almost parallel at lower frequencies and show some deviations at higher frequencies. This finding can be explained by the increasing distance to the reference stimulus. Two subjects in particular showed inconsistencies for higher test frequencies (see Figure 3). The inter-individual standard deviations increase with increasing frequency. The variations are independent of vibration magnitude and comparable to the results shown in Figure 4; they will not be plotted here for a better overview. The averaged contours increase depending on the frequency from $10 \mathrm{~Hz}$ to $250 \mathrm{~Hz}$. The slope is shallow up to $100 \mathrm{~Hz}$ and steepens for higher frequencies 


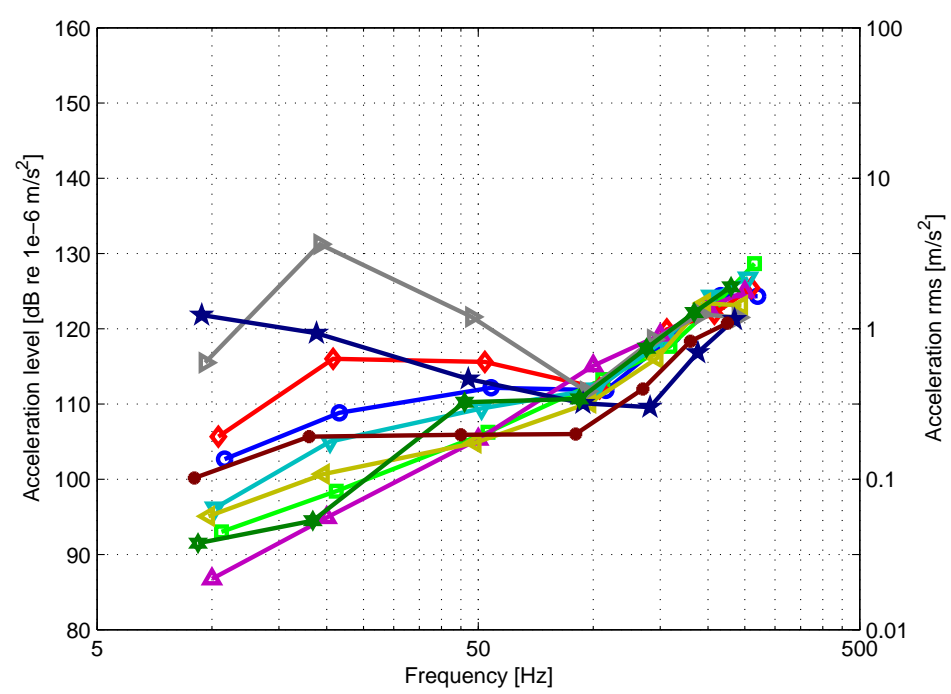

Fig. 2. Individual equal intensity contours for vertical sinusoidal whole-body vibrations for all subjects using a reference frequency of $100 \mathrm{~Hz}$ with an acceleration level of $110 \mathrm{~dB}$.

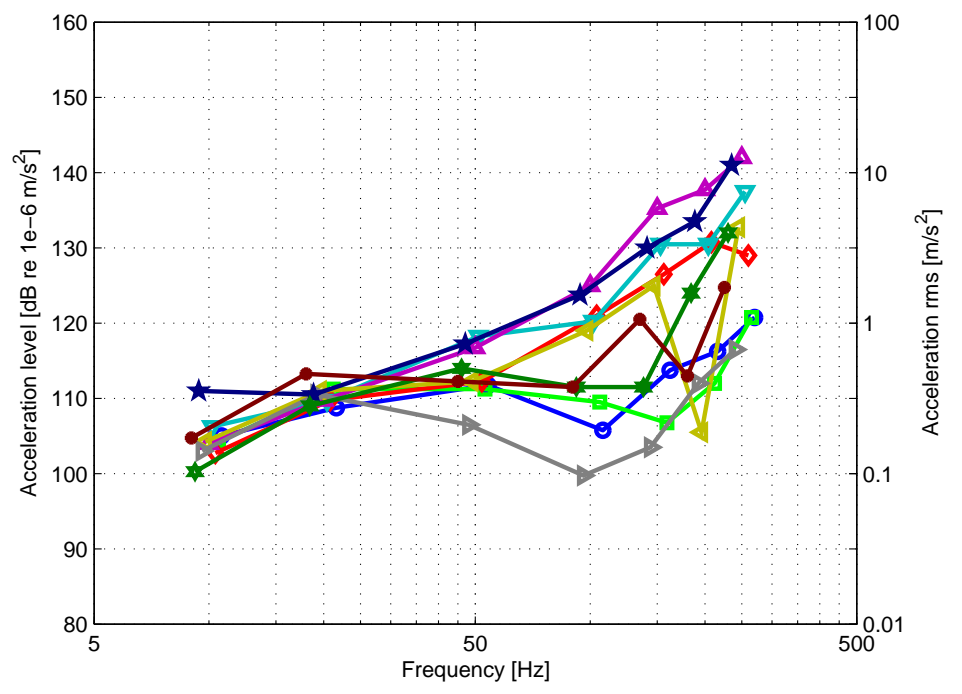

Fig. 3. Individual equal intensity contours for vertical sinusoidal whole-body vibrations for all subjects using a reference frequency of $20 \mathrm{~Hz}$ with an acceleration level of $110 \mathrm{~dB}$. 


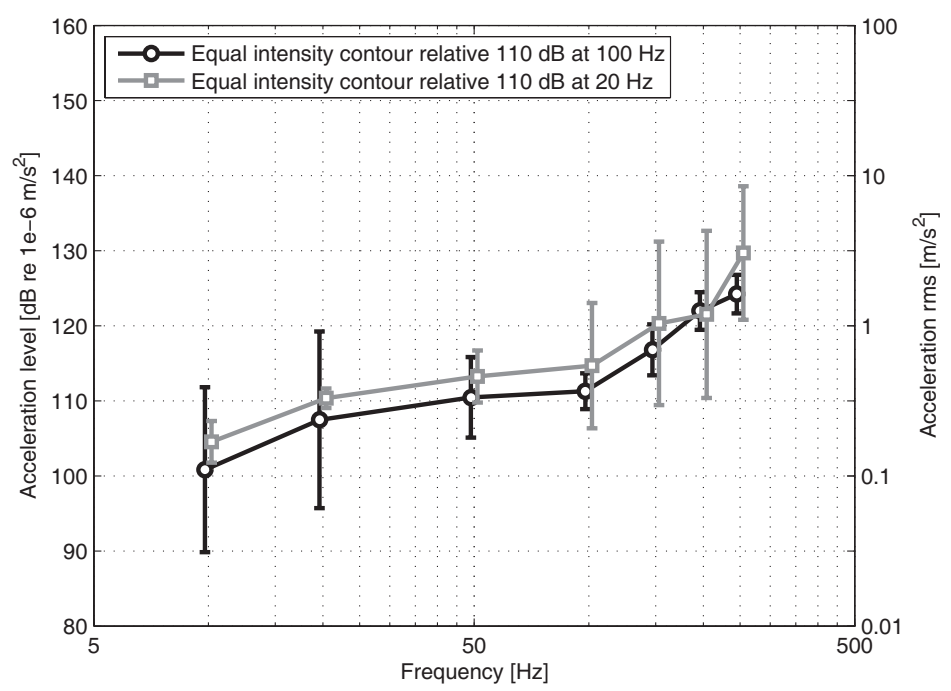

Fig. 4. Comparison between averaged equal intensity contours for vertical sinusoidal whole-body vibrations using a reference frequency of $20 \mathrm{~Hz}$ and $100 \mathrm{~Hz}$ with an acceleration level of $110 \mathrm{~dB}$.

up to $250 \mathrm{~Hz}$. The curves increase above $100 \mathrm{~Hz}$ with approximately $9 \mathrm{~dB}$ per octave. The general shape is comparable with the averaged perception threshold, which is also plotted in Figure 5 for comparison.

Few studies have investigated equal perception contours for low magnitudes of vibration and/or a broad frequency range. Bellmann [6] measured an equal intensity contour between $5 \mathrm{~Hz}$ and $80 \mathrm{~Hz}$ using an adaptive two-interval forced choice procedure. A reference frequency of $20 \mathrm{~Hz}$ at $100 \mathrm{~dB}$ was used, which corresponds to the weakest reference stimuli of the present study. The resulting contour is plotted in Figure 5 for comparison and shows reasonable agreement.

Morioka \& Griffin [12] used magnitude estimation to determine judgements of discomfort caused by vertical vibrations over a wide frequency and dynamic range. Equal (dis-)comfort contours were then calculated from the data. The resulting curves are plotted in Figure 5 for comparison. Note that the bottom and top contour in the plot do not predominantly represent measured vibrations but are determined by extrapolation. The contours show a similar tendency as in the present study, but no pronounced break at $100 \mathrm{~Hz}$. The data from Morioka \& Griffin [12] confirm that the contours are similar to the perception threshold for low sensation magnitudes. However, Morioka \& Griffin [12] found a dependence of the magnitude on the shape of the equal comfort contours. This result differs somewhat from the present findings but might be explained by the different experimental question (discomfort versus intensity) and the solely low 


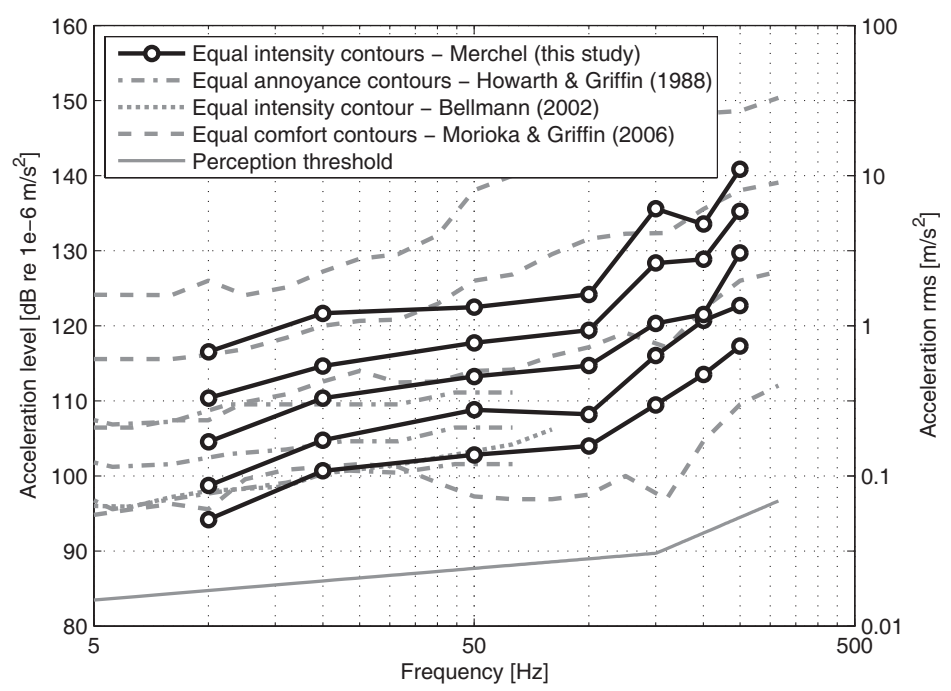

Fig. 5. Comparison of equal perception contours for sinusoidal vertical whole-body vibrations from different laboratories.

acceleration levels used in the present study. The results at low magnitudes in the present study deviate in general from literature using higher accelerations (e.g., health risk, annoyance or discomfort curves; see Griffin [2] for an overview), which show steeper slopes.

Earlier data can be found in Howarth \& Griffin [13], who used an acoustical reference stimulus $(1 / 3$ octave band noise centered at $1 \mathrm{kHz})$ to measure annoyance caused by vibration using a method of magnitude estimation. Only frequencies up to $63 \mathrm{~Hz}$ were measured with a small dynamic range. Interestingly, the resulting annoyance contours are consistent with the equal intensity contours from the present study as illustrated in Figure 5.

Merchel et al. [1] hypothesized that cross-modally matched vibrations with tones from equal loudness contours might be similar to equal intensity contours for whole-body vibrations like the ones measured in the present study. Figure 6 shows the results from both studies for comparison. The data is consistent between experiments. This finding suggests that equal intensity contours could be used to predict cross-modal matching between the loudness of sounds and the perceived intensity of whole-body vibrations. Most participants reported that intensity matching within a modality, which reduces experimental time and complexity, is a much easier task than matching across modalities.

Merchel et al. [1] found that an increase of 20 phon in the loudness level resulted in a $5 \mathrm{~dB}-6 \mathrm{~dB}$ increase in the matched acceleration level (at loudness levels greater than 40 phon). A 20 phon increase in the loudness level corresponds to 


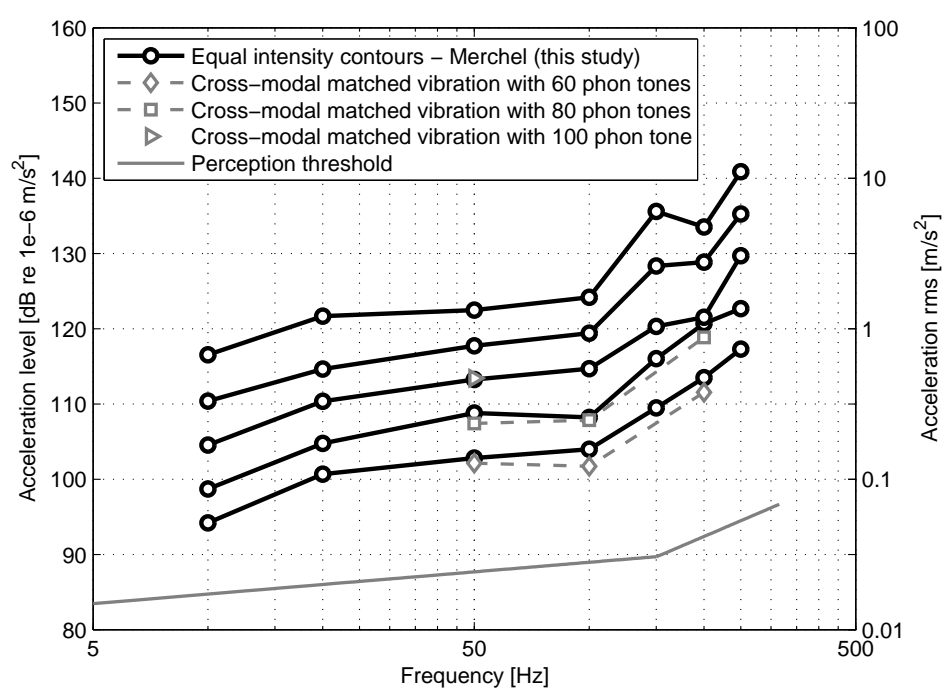

Fig. 6. Comparison of equal intensity contours for sinusoidal vertical whole-body vibrations (present study) with cross-modally matched vibrations with tones from equal loudness contours [1].

a quadruplication of perceived loudness. It might thus be assumed that the perceived intensity of sinusoidal vertical whole-body vibrations is approximately doubled through a $2.5 \mathrm{~dB}-3 \mathrm{~dB}$ increase in acceleration level. This trend may only be true for the investigated frequency and dynamic range and could be tested with a magnitude estimation experiment in a further study.

\section{Summary and Outlook}

In this study, contours of equally perceived intensity were determined for vertical sinusoidal whole-body vibrations. The following results were obtained:

- The shapes of the equal intensity contours in the present study show reasonable agreement with the contours from other studies despite the use of different methodologies and experimental questions.

- Small intra-individual and large inter-individual variations were observed.

- Contours from cross-modally matched vibrations with tones from equal loudness contours show a similar shape if compared to the measured equal intensity contours for whole-body vibrations of the present study. 
Future research should investigate even lower vibration magnitudes than the present study. Another open question is the variability between subjects, which remains to be explained.

\section{Acknowledgements}

The authors wish to thank Prof. U. Jekosch for her support and informative discussions.

\section{References}

1. Merchel, S. and Altinsoy, M.E.: Cross-Modality Matching of Loudness and Perceived Intensity of Whole-Body Vibrations, Proceedings of HAID'10, Copenhagen, Denmark (2010)

2. Griffin, M.J.: Handbook of Human Vibration, Academic Press, London (1990)

3. Miwa, T.: Evaluation Methods for Vibration Effect, Part 1: Measurements of Threshold and Equal Sensation Contours of Whole-Body for Vertical and Horizontal Vibrations, Industrial Health 5, pp. 183-205 (1967)

4. Parsons, K.C. and Griffin, M.J.: Whole-Body Vibration Perception Thresholds, J. Sound Vib. 121, pp. 237-258 (1988)

5. Morioka, M., Griffin, M.J.: Absolute Thresholds for the Perception of Fore-and-Aft, Lateral, and Vertical Vibration at the Hand, the Seat, and the Foot, J. Sound Vib. 314, pp. 357-370 (2008)

6. Bellmann, M.: Perception of Whole-Body Vibrations: From Basic Experiments to Effects of Seat and Steering-Wheel Vibrations on the Passenger's Comfort Inside Vehicles, Ph.D. Thesis, Carl von Ossietzky Universitt Oldenburg (2002)

7. Stamm, M., Altinsoy, M.E. and Merchel, S.: Frequenzwahrnehmung von Ganzkrperschwingungen im Vergleich zur auditiven Wahrnehmung I und II, Proceedings of DAGA'10, Berlin, Germany (2010)

8. Merchel, S., Leppin, A. and Altinsoy, M.E.: The Influence of Whole Body Vibrations on Loudness Perception, ICSV 16, Krakw, Poland (2009)

9. International Organization of Standardisation: ISO 2631-2:1989 Evaluation of Human Exposure to Whole-Body Vibration - Part 2: Continuous and Shock-Induced Vibrations in Buildings (1 Hz to $80 \mathrm{~Hz}$ ), Geneva (1989)

10. International Organization of Standardisation: ISO 2631-2:2003 Evaluation of Human Exposure to Whole-Body Vibration - Part 2: Vibrations in Buildings $(1 \mathrm{~Hz}$ to $80 \mathrm{~Hz}$ ), Geneva (2003)

11. Altinsoy, M.E. and Merchel, S.: BRTF - Body Related Transfer Functions for Whole-Body Vibration Reproduction Systems, DAGA, Rotterdam, Netherlands (2009)

12. Morioka, M., Griffin, M.J.: Magnitude-Dependence of Equivalent Comfort Contours for Fore-and-Aft, Lateral and Vertical Whole-Body Vibration, J. Sound Vib. 298, pp. $755-772$ (2006)

13. Howarth, H.V.C., Griffin, M.J.: The Frequency Dependence of Subjective Reaction to Vertical and Horizontal Whole-Body Vibration at Low Magnitudes, J. Acoust. Soc. Am., 83, (4), pp. 1406-1413 (1988) 